The Astrophysical Journal, 189: 459-462, 1974 May 1

(C) 1974. The American Astronomical Society. All rights reserved. Printed in U.S.A.

\title{
ON THE USE OF MEAN ESCAPE PROBABILITIES TO SOLVE TRANSFER PROBLEMS IN NEBULAE
}

\author{
A. P. Bernat And R. R. Robbins \\ University of Texas at Austin \\ Received 1973 July 20; revised 1973 November 26
}

\begin{abstract}
Results from an accurate numerical solution are compared with the solutions predicted by one particular formulation of mean escape probabilities. It is found that the latter do not predict numerical solutions well and that it is difficult to determine a priori how to improve the agreement. Solution of transfer problems by mean escape probabilities seems consistently to underestimate the optical depth in a transfer problem and hence the density. Mean escape probabilities can be extremely useful, however, in solving additional "nearby" cases when one solution is known to high accuracy; in sample calculations by the authors, problems previously requiring 4 hours on the computer were solved in 45 seconds, a factor of approximately 300 in computer time!

Subject headings: nebulae - radiative transfer
\end{abstract}

\section{INTRODUCTION}

The solution of the equation of radiative transfer for realistic nebular problems generally demands considerable man and computer time. In an attempt to simplify such problems, numerous authors (see, e.g., Capriotti 1965; Cox and Mathews 1969) have employed the conceptually much simpler formulation of mean escape probability. An expression is derived that depicts the probability of escape of a single photon emitted at some point in the nebula, and then a geometrical averaging over photons emitted from all parts of the nebula gives an average probability of escape which is assumed to apply to all photons.

The attraction of this method is its computational simplicity: problems which might require months to program and solve on a large computer may be reduced to a few hours on a desk calculator. However, up until now this approach has been applied only to problems that were intractable to more rigorous solutions, so that a quantitative estimate of its validity is lacking. The purpose of this paper is to examine one particular formulation of mean escape probabilities and to compare the results it gives with the results that are obtained by solving an identical transfer problem by rigorous transfer theory.

Thus, in $\S$ II we calculate mean escape probabilities for a specific nebular model, using the formulation of Capriotti (1965); in $\S$ III, we compare the mean escape predictions with the results from numerical solutions of an exact formulation of the same transfer problem, and conclude that mean escape probabilities are not particularly reliable approximations to rigorous solutions. In the final section we discuss one extremely productive use to which mean escape probabilities can be put, namely, the solution of "nearby" transfer problems quickly and accurately when one particular case has already been solved in detail.

\section{CALCULATION OF MEAN ESCAPE PROBABILITIES}

We shall use for our model of a nebula a spherical distribution of gas differentially expanding with a velocity distribution $v(r)=k r$, where $r$ is the distance from the center of the nebula. A photon emitted in an optically thick line from some point in the nebula will see an optical depth (see Robbins 1968) of

$$
\tau=\frac{\tau_{0}}{a} \int_{x}^{x-a s / R} \exp \left(-w^{2}\right) d w,
$$

where $s$ is the distance from the point of photon creation to the edge of the nebula, $\tau_{0}$ is the optical depth in the center of the line for a stationary nebula, and $a$ is the ratio of the velocity of expansion at the outer edge of the nebula $v(R)$ to the thermal velocity. We have here assumed complete redistribution in frequency according to the Doppler mechanism.

Following the mean escape probability formalism employed by Capriotti (1965), we shall assume that a photon seeing an optical depth less than some critical optical depth $\tau_{c}$ will escape, whereas the photon will be absorbed if equation (1) gives $\tau>\tau_{c}$. We have set $\tau=\tau_{c}$ in equation (1) and solved for a critical frequency $x_{c}$. A negative solution corresponds to $-\left|x_{c}-a s\right| R \mid=x_{2}$. If $x_{c}$ is negative, then all photons are assumed to escape. An averaged escape probability from the point $r$ is then given by the integral over all directions of emission

$$
\begin{aligned}
\epsilon(r)= & \frac{1}{4 \pi R^{2}} \int_{4 \pi} R^{2} d \Omega \\
& \times\left(\frac{1}{\sqrt{ } \pi} \int_{-\infty}^{x_{2}} e^{-w^{2}} d w+\frac{1}{\sqrt{ } \pi} \int_{x_{c}}^{\infty} e^{-w^{2}} d w\right),
\end{aligned}
$$

and the mean escape probability of the configuration is then given by averaging over the volume

$$
\epsilon=\left(\frac{4}{3} \pi R^{3}\right)^{-1} \int_{4 \pi} d \Omega \int_{0}^{R} \epsilon(r) r^{2} d r .
$$

Table 1 gives our computations of $\epsilon$ for various optical depths and several expansion velocities, assuming that 
TABLE 1

Mean Escape Probabilities for Expanding Uniform SPHeres

\begin{tabular}{|c|c|c|c|c|c|c|}
\hline \multirow[b]{2}{*}{$\tau$} & \multicolumn{2}{|c|}{$a=1$} & \multicolumn{2}{|c|}{$a=3$} & \multicolumn{2}{|c|}{$a=5$} \\
\hline & $\tau_{c}=1.0$ & $\tau_{c}=1.3$ & $\tau_{c}=1.0$ & $\tau_{c}=1.3$ & $\tau_{c}=1.0$ & $\tau_{c}=1.3$ \\
\hline
\end{tabular}

photons are created at a uniform rate throughout the nebula (the assumption that all photons are created in the center causes very little change in the values). All previous $\epsilon$ calculations have assumed that $\tau_{c}=1$ is the critical optical depth at which escape should be assumed. This is an average optical depth at which a photon first "sees" the edge of the nebula, however, and there is of course a distribution of escape probability with optical depth. We have included some calculations for other values of $\tau_{c}$ to indicate the degree to which the mean escape probabilities are sensitive to this number. The critical optical depth $\tau_{c}$ will presumably never be much different from unity in a real physical situation; however, it could be used as an adjustable parameter to make mean escape computations fit the results of a particular transfer solution.

It should be noted that although our formulation is identical to that of Capriotti (1965), our computed values for $\epsilon$ when $\tau_{c}=1$ do not agree with his table 3 values. Since the earlier calculations were slide-rule computations using low-order quadratures, it is our opinion that the present calculations are somewhat more accurate.

Given a mean escape probability $\epsilon_{n}$ for the $n$th line in a series of optically thick transitions (for example, the Lyman lines of hydrogen), the emergent intensity of the line is given by

$$
I_{n}=\epsilon_{n}\left[C_{n}+\sum_{m=n}^{N} P(n, m) A_{m}\right],
$$

where $C_{n}$ equals the rate at which $n$th line photons are created in the nebula by capture-cascade (case A) processes. The $A_{m}$ are the number of absorptions the photons of the $m$ th line suffer in attempting to escape the nebula, and the $P(n, m)$ are probability factors computed from the Einstein $A(n, m)$ which express the likelihood that a photon is either scattered or degraded to other wavelengths upon absorption. Equation (4) simply states that when a series of transitions is optically thick, absorptions of a higher line in the series (e.g., L $\delta$ ) may create additional $\mathrm{L} \beta$ photons which attempt to escape the nebula. Mean escape probability theory relates the number of absorptions to the emergent intensity through the relation

$$
I_{m}=\frac{\epsilon_{m}}{1-\epsilon_{m}} A_{m}
$$

Again, these formulae are all taken from Capriotti (1965).

\section{COMPARING $\epsilon$-PREDICTIONS WITH RESULTS FROM RIGOROUS SOLUTIONS}

Robbins (1968) and Robbins and Bernat (1974) have formulated integral transfer equations expressing the escape of radiation in the principal series of the triplets and singlets of neutral helium and have carried out extensive numerical solutions of these equations. The authors have used the existing computer programs from these studies to numerically solve single-line transfer problems for comparison with mean escape probability predictions. Specifically, solutions have been carried out for the $\lambda 584\left(1^{1} S-2{ }^{1} P\right)$ transition of $\mathrm{He} \mathrm{I}$. Photons in this line, attempting to escape a nebula of optical thickness $\tau$, have a probability $\alpha=$ 0.9988 of being simply scattered upon absorption by a helium atom in its ground state, and a probability 1 $\alpha=0.0012$ of being destroyed and degraded to other wavelengths. In figure 1 we show the results of these calculations, where the quantity plotted is $R(\tau)=$ $I(\lambda 584, \tau) / I(\lambda 584, \tau=0)$.

We have also plotted in figure 1 the emergent intensities that are predicted for $R(\tau)$ by $\epsilon$-theory. The plotted numbers are the solutions of equations (4) and (5) above, with $N=n$. Several interesting conclusions can immediately be drawn from this figure. First, it is clear that no single value of $\tau_{c}$ satisfactorily reproduces the transfer equation results for all values of $\tau$. For small optical depths, $\tau_{c}=1.2$ looks appropriate; but for most optical depths between 10 and $100, \tau_{c}=1.35$ looks better. Second, mean escape probabilities computed on the assumption that $\tau_{c}=1$ turn out to be systematically too small and hence systematically 


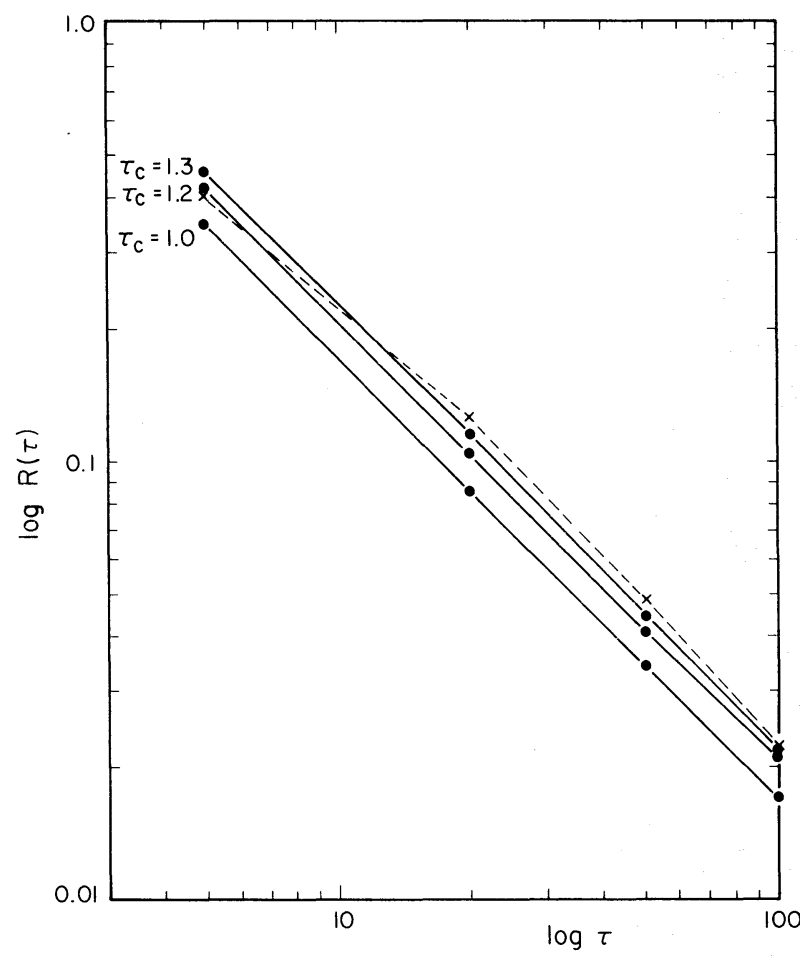

FIG. 1.-A comparison of the intensity of $\lambda 584$ obtained from numerical transfer solutions and from mean escape probability formulae. The solid lines show the mean escape probability predictions for varying critical optical depths and are to be compared with the exact solution, given by the dotted line.

underestimate $R$. As a consequence, they will systematically underestimate the optical depth that would be deduced from the observed line ratios. Since $\tau_{0}=$ $\kappa_{0} N D$, where $\kappa_{0}$ is the line absorption coefficient, $N$ is the space density of absorbers, and $D$ is the dimension of the nebula, optical-depth uncertainties translate directly into errors in the derived densities.

The optical depth and density of absorbers are derived by comparing observed and computed $R$ 's, and a small change in $R$ may make a large difference in the derived density. Thus, the differences in figure 1 are quite significant. As an example, in the helium triplets $\lambda 7065$ is the most reliable indicator of the number of absorbers in the metastable $2{ }^{3} S$ level, as it is formed by the degradation of $\lambda 3889 \mathrm{He}$ I photons. As $\tau(3889)$ changes from 0 to 20 , the intensity of $\lambda 7065$ changes from 0.66 to 2.04 ; but a further increase in $\tau(3889)$ to 75 changes $I(7065)$ to only 2.63 . The exact variation of density with line intensity will depend upon the conversion probabilities and the optical depths in each particular problem; in general, the dependence is nonlinear and can be dramatic. If one were interested only in reproducing the intensities in a given problem, then the use of mean escape probabilities would represent less risk than in the case where the desired information was the astrophysically more significant density.

The inaccuracies involved in assuming that $\tau_{c}=1$ are smallest when the optical depth is small; but for problems involving optical depths from 5 to 100 (the limit of the present calculations), $\tau_{c} \simeq 1.3$ would give mean escape probabilities that more closely approximated the results from transfer solutions which assume complete redistribution upon scattering.

It is possible to "adjust" $\tau_{c}$ until it reproduces a given transfer problem exactly. However, this adjustment will depend upon such factors as the geometry of the nebula, the distribution of the absorbing atoms, and the type of scattering; without an a priori physical or mathematical basis for this adjustment, it is necessary to solve the problem anyway to see what the adjustment should be. Thus, there is effectively no gain from using mean escape probabilities. A plausible methodology for adjusting $\tau_{c}$ 's or computing better $\epsilon$ 's for an arbitrary transfer problem (such that it would not be necessary ever to solve the equation of transfer) is not readily apparent.

We have also investigated the dependence upon the scattering parameter $\alpha$ by numerically solving the exact transfer problem for the $\lambda 584$ line but allowing $\alpha$ to vary. This gives an artificial problem to some extent, but at least it is one in which all other factors are held strictly constant. We have varied $\alpha$ over a range from 0.01 to 0.99 , and then we have determined the value that $\epsilon$ must have to reproduce the numbers from the transfer solution. Such effective mean escape probabilities were found to have very little variation with $\alpha$. However, the emergent intensities are so sensitive to small changes in $\epsilon$ that even a small variation of $\epsilon$ with $\alpha$ may be unacceptable. By manipulation of equations (4) and (5) above, it is simple to demonstrate that

$$
R_{\epsilon}=\frac{\epsilon}{1-\alpha(1-\epsilon)}
$$

Thus, errors in $\epsilon$ are multiplied by $\alpha$ in computing the line intensities and (as discussed above) errors in line intensities are multiplied again when densities are derived. Thus, effective $\epsilon$ 's should be derived for the case of largest $\alpha$ that will be considered; if they are satisfactory when $\alpha$ is near 1 , then they will be even more accurate for $\alpha$ near zero.

The main conclusion of this section is that mean escape formulae such as equations (1)-(6) have some usefulness in predicting nebular spectra, but probably cannot do so with the high accuracy required to deduce optical depths and densities accurately, unless some method is found for adjusting the critical optical depth in the line, $\tau_{c}$, in advance, for arbitrary transfer problems.

\section{THE SOLUTION OF "NEARBY" TRANSFER PROBLEMS}

In spite of their negative conclusions, the previous sections do in fact suggest one extremely valuable use for mean escape probabilities. If a transfer problem has been solved to high accuracy by the use of numerical methods, it is possible to choose a set of empirical (or "effective") mean escape probabilities which will reproduce the computed spectrum to very high accuracy. 
These $\epsilon_{\text {eff }}$ 's should then be usable to reproduce transfer solutions similar to the first. Commonly, a transfer problem must be fully solved many times, for each variation and combination of the input parameters or boundary conditions to the problem. If the problem could be solved only once and then effective $\epsilon$ 's used to carry out all the other solutions, considerable savings in computer time would result.

To test the usefulness of effective $\epsilon$ 's in this fashion, the authors used the results of Robbins and Bernat (1974), a complete set of helium-singlet transfer solutions for three electron temperatures and various expansion velocities and optical depths. The combinations of parameters gave about 40 cases to examine. We took the transfer intensities for $T_{e}=20,000^{\circ} \mathrm{K}$ and computed $\epsilon_{\text {eff }}$ 's for them, and then used these mean escape probabilities to compute all the various cases for electron temperatures of $5,000^{\circ}$ and $10,000^{\circ} \mathrm{K}$. The results were spectacularly successful: the effective mean escape probabilities reproduced the transfer solution intensities exactly in all lines of the helium spectrum for $10,000^{\circ} \mathrm{K}$, and for $5,000^{\circ} \mathrm{K}$ the only deviations were on the order of a few percent in some of the higher lines of the principal series-lines too weak to be observed by present techniques. The difference in computer time between the two situations is to be emphasized. In this particular situation approximately 4 hours' worth of computer solutions were duplicated to four significant figures in a total of 45 seconds! As a further illustration of their usefulness, we note that improved case A intensities for the helium singlets have been calculated by Brocklehurst (1972); ordinarily, this would necessitate redoing the entire 4-hour sequence of transfer calculations to get updated intensities with optical depth present. With mean escape probabilities, such updated numbers are only 45 computer seconds away.

We take pleasure in thanking Professor Eugene Capriotti for helpful discussions on this work.
Brocklehurst, M. 1972, M.N.R.A.S, 157, 211.

Capriotti, E. R. 1965, Ap. J., 142, 1101.

Cox, D. P., and Mathews, W. G. 1969, Ap. J.,155, 859.

\section{REFERENCES}

Robbins, R. R. 1968, Ap. J., 151, 511.

Robbins, R. R., and Bernat, A. P. 1974, Ap. J., 188, in press. 